\title{
First International Workshop of the Tiss.EU Project: Rights and Entitlements in Human Tissue and Cells
}

\author{
Hannover, 28.-29. November 2008
}

\author{
Katharina Beier
}

Online publiziert: 27. Mai 2009

(C) Die Autor(en) 2009. Dieser Artikel ist auf Springerlink.com mit Open Access verfügbar.

Mit dem Workshop zum Thema „Rights and Entitlements in Human Tissue and Cells“ (organisiert von Nils Hoppe, AG Medizinrecht \& Bioethik der Universität Hannover), wurde am 28. und 29. November 2008 der Auftakt zu insgesamt neun internationalen Expertentreffen gegeben, die im Rahmen des Tiss.EU Projektes (http://www.tisseu.org) bis Anfang 2011 stattfinden. Das seit April 2008 im 7. Rahmenprogramm laufende EU-Projekt beschäftigt sich mit Fragen der ethischen und rechtlichen Regulierung der Forschung mit menschlichen Geweben und Zellen in den 27 EU-Staaten und der Schweiz. Die Koordination des Projekts liegt bei Dr. Christian Lenk und Prof. Dr. Claudia Wiesemann (Abt. Ethik und Geschichte der Medizin, Universität Göttingen) in Zusammenarbeit mit Dr. Nils Hoppe.

Der Workshop in Hannover widmete sich schwerpunktmäßig der Frage nach Rechten und Ansprüchen im Umgang mit menschlichen Geweben und Zellen. Dabei waren neben Experten aus Wissenschaft und Praxis insbesondere Juristen aus der Schweiz, Österreich und Deutschland geladen, um über den rechtlichen Rahmen der Gewebeforschung in diesem ausgewählten Länderkreis zu informieren. In seinem Impulsreferat stellte der Jurist Nils Hoppe drei zentrale Problemkomplexe der Gewebeforschung heraus:

- Klärungsbedürftig ist erstens das Verhältnis von therapeutischer Nutzung und Forschung an menschlichen Geweben und Zellen. Die EU-Richtlinie zur therapeutischen Gewebenutzung bildet dafür einen wichtigen Anhaltspunkt, insofern sie die noch ausstehende Regelung der Gewebeforschung mit hoher Wahrscheinlichkeit beeinflussen wird.

- Infrage steht zweitens der rechtliche Status von Zellen und Gewebe nach der Entnahme aus dem menschlichen Körper. Obwohl dieser in den europäischen Ländern als res extra commercium gilt, trifft dies nicht gleichermaßen für vom Körper abgetrennte Materialien zu. So besitzen technisch veränderte Gewebe in Deutschland zum Beispiel den Status eines medizinischen Produkts.

- Eng damit verbunden ist drittens die Frage nach den Verfügungsrechten über menschliches Gewebe. Im Zentrum steht hier der augenfällige Widerspruch, dass das Verbot der

K. Beier $(\bowtie)$

Abt. Ethik und Geschichte der Medizin, Georg-August-Universität Göttingen, Humboldtallee 36

37073 Göttingen, Deutschland

E-Mail: katharina.beier@medizin.uni-goettingen.de 
Kommerzialisierung von Körpermaterialien praktisch nur den Spender betrifft, während Dritte diese durchaus zu gewinnbringenden Zwecken nutzen können.

Dass das Recht insbesondere auf die von der Gewebeforschung aufgeworfenen ethischen Fragen reagieren muss, machte der Jurist José Miola (Universität Leicester) in seiner Auseinandersetzung mit den Konsensbestimmungen des britischen Human Tissue Act deutlich. Dabei kritisierte Miola, dass in dem 2004 - in Reaktion auf vorangegangene Gewebeskandale - verabschiedeten Gesetz der Schutz der Gewebespender in mehrfacher Hinsicht unbefriedigend geblieben sei. So habe die gegenüber dem Gesetz kritisch eingestellte medizinische Profession eine Aufweichung der ursprünglich vorgesehenen spezifischen Konsensregelung zugunsten einer, ,general consent"-Lösung erwirkt und zudem durchgesetzt, dass bereits existierende Gewebesammlungen weiterhin ohne expliziten Konsens der Spender genutzt werden könnten.

Im Unterschied zu Großbritannien wiesen die Juristen Prof. Dr. Jürgen Simon (Universität Lüneburg), Dr. Bianka Dörr (Universität Zürich) und Markus Kastelitz (Universität Hannover) als Vertreter des für den Workshop gesetzten Länderschwerpunktes darauf hin, dass die Nutzung menschlicher Gewebe und Zellen in Deutschland, Österreich und der Schweiz durch eine äußerst fragmentierte Gesetzgebung geregelt ist, die sich auf Transplantations-, Transfusions-, Arzneimittel- und Bestattungsgesetze erstreckt. Wie Simon betonte, sind mit diesem Ansatz ebenfalls Regelungslücken verbunden, die vor allem die Forschung mit menschlichem Gewebe betreffen: So sei es etwa problematisch, die in deutschen Operationssälen anfallenden Gewebe weiterhin als „Reste“ zu deklarieren, wenn diese zum Aufbau systematischer Gewebesammlungen genutzt werden könnten. Auch in Österreich bestehe diesbezüglich wenig Klarheit. Dem Argument, die bei Operationen rechtmäßig entnommenen Körpermaterialien seien das Eigentum des Krankenhauses, steht laut Markus Kastelitz das Urteil des Österreichischen Verfassungsgerichts entgegen, dass die Nutzung von Geweben ohne vorherige Zustimmung des Verstorbenen unzulässig sei. Was dies für die Forschung mit menschlichen Geweben bedeutet, vermag auch das ausschließlich therapeutische Anwendungen erfassende Gewebesicherheitsgesetz nicht deutlicher zu machen. Simon und Kastelitz plädierten daher für ein übergreifendes Gewebeforschungsgesetz, das insbesondere die Entnahme von Geweben bei lebenden Spendern regeln solle. Mit einem für 2009 erwarteten umfassenden Humanforschungsgesetz geht die Schweiz über diese Forderung bereits hinaus. Für eine Einschätzung, inwieweit damit auch die Gewebeforschung befriedigend geregelt wird, ist es laut Bianka Dörr jedoch zu früh. Mit Blick auf den britischen Human Tissue Act meldete Miola allerdings Zweifel daran an, dass ein übergreifendes Gewebegesetz gegenüber einer fragmentierten Gesetzgebung die bessere Lösung sei.

Einigkeit besteht in den drei mehrheitlich deutschsprachigen Ländern grundsätzlich darüber, dass die Nutzung menschlicher Gewebe eine Frage persönlichkeitsrechtlicher Natur darstellt, die Person aber, sobald die Gewebe von ihrem Körper getrennt vorliegen, faktisch ein eigentumsähnliches Verfügungsrecht über diese besitzt. Dieser Dualismus - so Bianka Dörr - provoziere jedoch die Frage, wie mit der Transformation von Geweben und Zellen in Quasi-Eigentum zugleich ein Verlust von Persönlichkeitsrechten verhindert werden könne. Trotz der in Deutschland, Österreich und der Schweiz in Teilen übereinstimmenden Rechtslage kamen während des Workshops auch Unterschiede zur Sprache. Diese zeigten sich etwa bei der Transplantationsgesetzgebung. In der Diskussion waren sich die Teilnehmer allerdings darüber einig, dass diese zwar die Nutzung von Geweben zu Therapiezwecken beeinflusse, jedoch kaum Auswirkungen auf die zukünftige Regelung der Forschung mit menschlichem Gewebe haben werde. 
Dass für die Nutzung von Geweben zu Forschungszwecken rechtlicher Regelungsbedarf besteht, machten insbesondere zwei Beiträge aus der Praxis deutlich. So wies Michael Harder - als Geschäftsführender Direktor der 2006 aus der Medizinischen Hochschule Hannover ausgegründeten Corlife GbR, die Produkte für die cardiovaskuläre Chirurgie herstellt - auf den ungeklärten Rechtsstatus der von Corlife aufbereiteten Bindegewebe von gespendeten Herzklappen hin. Da Forschungs- und Entwicklungsaktivitäten in Deutschland nicht durch den informierten Konsens gedeckt seien, importiere Corlife derzeit sicherheitshalber Herzklappen aus den USA. Der Vertreter des Verbands der Universitätsklinika Deutschland e.V., Ralf Heyder, wandte sich in seinem Vortrag gegen eine Dämonisierung von Profit als solchem. Da sämtliche Nutzung von Geweben Geld koste, gelte es vielmehr, legitime Formen der Ökonomisierung von illegitimer Kommerzialisierung zu unterscheiden. Während es ethisch unzulässig sei, Profit aus der Bedürftigkeit von Patienten zu schlagen, könnten andererseits durch eine entsprechende administrative Preisregulierung wünschenswerte Effekte wie Innovation oder Effektivität befördert werden. Vor diesem Hintergrund plädierte Heyder zum Beispiel für eine angemessene Erstattung von Transplantationskosten gegenüber Kliniken, um den Anreiz zur Organ- bzw. Gewebeentnahme zu erhöhen.

Mit dem Beitrag des Juristen Marcello Corrales (Universität Hannover) zur europäischen Datenbankrichtlinie wurde deutlich, dass auch das EU-Recht mit der Gewebeforschung und ihren technischen Begleitumständen zu ringen hat. So werden etwa genetische Datenbanken von der EU-Richtlinie nicht eigens erfasst. Unter der Voraussetzung, dass auch für genetische Datenbanken intellektuelle Eigentumsrechte denkbar sind, warnte Corrales davor, dass Forschern der Zugang zu Daten erschwert bzw. dem „kreativen Investor“ der Datenbank sogar ein faktisches Monopol über bestimmte Daten eingeräumt werden könnte. Auch bestünden gewisse Spannungen zu dem mit der Datenschutzrichtlinie eingeräumten Recht des Individuums, Zugang zu seinen persönlichen Daten zu haben.

Die Verbindung von Länderberichten mit Beiträgen zu Gewebe nutzenden Forschungsprojekten sowie Vorträgen zu technischen und ökonomischen Aspekten der Gewebeforschung erwies sich als äußerst fruchtbar, um die damit verbundenen ethischen und rechtlichen Herausforderungen während des zweitägigen Workshops intensiv zu diskutieren. Dabei verstärkte sich bei allen Beteiligten der Eindruck, dass die Gewebeforschung Fragen aufwirft, auf die das nationale aber auch das EU-Recht derzeit nur begrenzt Antworten liefern. Die zwischen Deutschland, Österreich und der Schweiz aufgezeigten Übereinstimmungen, die nicht zuletzt daher rühren, dass selbst die Schweiz als Nicht-EU-Mitglied sich an EU-Richtlinien orientiert, bestätigten indessen den Ansatz des Tiss.EU-Projekts, im Rahmen weiterer Workshops zu untersuchen, inwieweit sich diese Beobachtung auch auf andere Länderkreise, die durch eine ähnliche Rechtskultur verbunden sind, übertragen lässt. Zu dem Ziel, ein europaweites Expertennetzwerk zur Gewebeforschung aufzubauen, hat der Workshop einen wichtigen Beitrag geleistet und die Teilnehmer bekräftigten, ihre in Hannover begonnene Zusammenarbeit fortsetzen zu wollen.

Open Access: $\quad$ Dieser Artikel unterliegt den Bedingungen der Creative Commons Attribution Noncommercial License. Dadurch sind die nichtkommerzielle Nutzung, Verteilung und Reproduktion erlaubt, sofern der/die Originalautor/en und die Quelle angegeben sind. 